\title{
Whole-exome sequencing identifies mutated PCK2 and $H U W E 1$ associated with carcinoma cell proliferation in a hepatocellular carcinoma patient
}

\author{
YAN-XUAN LIU ${ }^{1 *}$, SHU-FANG ZHANG ${ }^{2 *}$, YING-HUA JI ${ }^{3}$, SHENG-JU GUO ${ }^{4}$, \\ GENG-FU WANG ${ }^{5}$ and GUANG-WEN ZHANG ${ }^{6}$ \\ ${ }^{1}$ Department of Genetic Disease, The First Affiliated Hospital of Xinxiang Medical University, Weihui 453100; \\ ${ }^{2}$ Central Laboratory, Affiliated Haikou Hospital Xiangya School of Medicine Central South University \\ (Haikou Municipal People's Hospital), Haikou, Hainan 570208; \\ Departments of ${ }^{3}$ Oncology, ${ }^{4}$ Pharmaceutics, ${ }^{5}$ Anesthesiology and ${ }^{6}$ Infectious Diseases, \\ The First Affiliated Hospital of Xinxiang Medical University, Weihui 453100, P.R. China
}

Received April 23, 2012; Accepted July 20, 2012

DOI: $10.3892 / \mathrm{ol} .2012 .825$

\begin{abstract}
Hepatocellular carcinoma (HCC) is diagnosed in more than half a million individuals worldwide every year. It is often invasive and metastatic, resulting in a poor prognosis. Our knowledge of the genomic alterations implicated in HCC initiation and progression is fragmentary, and few molecular alterations unique to $\mathrm{HCC}$ are known. We performed whole-exome sequencing for a pleomorphic cell-type HCC tissue and matched normal tissue, and uncovered seven non-synonymous somatic variants in SPATA21, PPCS, CDH12, OR1L3, PCK2, HUWE1 and PHF16. These variants were validated by PCR and sequencing, with the exception of that in PPCS. We further performed a bioinformatics analysis of the six validated variants. The results suggested that the function of the proteins of the three mutated genes, $P C K 2$, HUWE1 and PHF16, may be changed significantly. Among these genes, $P C K 2$, within the insulin signaling pathway, and $H U W E 1$, within the ubiquitin-mediated proteolysis pathway, may be essential for cell proliferation. These pathways are known to be important for hepatocarcinogenesis. Hence, we suggest that $P C K 2$ and $H U W E 1$ are associated with carcinoma cell proliferation in HCC.
\end{abstract}

Correspondence to: Dr Yan-Xuan Liu, Department of Genetic Disease, The First Affiliated Hospital of Xinxiang Medical University, 88 Jiankang Road, Weihui 453100, P.R. China

E-mail: liuyx999@126.com

*Contributed equally

Key words: hepatocellular carcinoma, exome sequencing, somatic mutation

\section{Introduction}

Hepatocellular carcinoma (HCC) is diagnosed in more than half a million individuals worldwide every year. Liver cancer is the fifth most common cancer in males and the seventh most common in females. Most of the burden of the disease (85\%) is borne in developing countries, with the highest incidence rates reported in regions where infection with hepatitis B virus (HBV) is endemic, including Southeast Asia and sub-Saharan Africa. Additional risk factors for HCC are alcohol, toxins, inlcuding aflatoxin, hemochromatosis, $\alpha 1$-antitrypsin deficiency and non-alcoholic fatty liver disease (NAFLD). HCC rarely occurs before the age of 40 years and reaches a peak at approximately 70 years of age. Rates of liver cancer among males are two to four times as high as the rates among females (1-5). Despite major efforts to improve the diagnosis and treatment of HCC, therapeutic options remain limited. Most patients, especially in Asia and sub-Saharan Africa, present at end stages of the disease or with underlying liver cirrhosis and consequently surgical options may no longer be indicated. Thus, there is a need for novel therapeutic agents and strategies. Despite its global significance, HCC is understudied compared with other major lethal types of cancer, and hence, our knowledge of the genomic alterations implicated in HCC initiation and progression is fragmentary. An improved understanding of the molecular genetic alterations specific to HCC may lead to the development of more efficient methods of prevention, early diagnosis and cure of this disease.

In this study, we carried out whole-exome sequencing using DNA obtained from HCC and matched normal tissue and found six previously unidentified variants in six genes. We further studied the molecular effects of these variants.

\section{Materials and methods}

Tissue and DNA preparation. HCC tissue was obtained from a 69-year-old male patient, who had chronic HBV infection 
Table I. Summary of exome sequencing.

\begin{tabular}{lccc}
\hline Item & HCC & Matched normal & Average \\
\hline Total bases sequenced (Gb) & 4.64 & 4.66 & 4.65 \\
Mean depth of target region (-fold) & 24 & 63 & 44 \\
Percentage of the targeted bases which were covered at least once (\%) & 98.4 & 98.8 & 98.6 \\
Percentage of the targeted bases which were covered sufficiently for variant calling (\%) & 87.6 & 96.6 & 92.1 \\
Percentage of genes having $>95 \%$ of their coding bases called (\%) & 70.4 & 83.5 & 77.0
\end{tabular}

HCC, hepatocellular carcinoma.

and lymph node metastasis. The matched normal tissue was obtained from a distance of 3-4 cm from the HCC. Informed consent for the research was obtained from the ethical committee of the Hospital. DNA of these tissues was extracted using the traditional phenol chloroform method.

Informed consent was obtained from the patient. The study was approved by the ethics committee of the First Affiliated Hospital of Xinxiang Medical University, Weihui, China.

Targeted sequence capture and sequencing. SeqCap EZ Human Exome Library version 2.0 (Roche Diagnostics, Mannheim, Germany; 44.1 Mb regions are covered by the probes. Cat no. 05860504001) was used for sequence capture according to the manufacturer's instructions. Genomic DNA $(10 \mu \mathrm{g})$ was used to prepare the library with the Truseq DNA Sample Prep kit from Illumina (Illumina, San Diego, CA, USA; Cat. no. FC-121-1001). Sequencing was performed at a concentration of $12 \mathrm{pM}$ on an Illumina Genome Analyzer IIx with paired-end 115 -bp reads.

The genomic DNA library preparation, targeted sequence capture and massively parallel sequencing were completed by Guangzhou iGenomics Co., Ltd. (Guangzhou, China).

Alignment, variants calling and quality control. The software BWA (version 0.5.9) (6) was used to align the paired-end reads to the reference human genome (hg19). After the alignment, PCR duplications were removed using the SAMtools software package (version 0.1.16) (7). Candidate somatic variants were identified with the VarScan 2 software (version 2.2.8) and filtered by the accessory script (fpfilter.pl, version 1.01) with default parameters (8). To qualify the identified somatic variants, all candidate variants were subjected to manual review using the Integrative Genomics Viewer (9). Common variants were excluded by filtering known germ-line variants in Ensembl (version 64, http://sep2011.archive.ensembl.org/ index.html) and an internal database composed of variants which occurred more than twice in 40 publicly available control genomes. A variant was noted if it was annotated as associated with a phenotype by Ensembl.

Bioinformatics analysis of non-synonymous somatic variants. The effects of the non-synonymous somatic variants were evaluated by bioinformatics analysis. The analysis of the chemical polarity and conservation, prediction of secondary structure and the domain of the proteins were performed. The conservation analysis was carried out by PhyloP (10) and
MutationTaster (11), the prediction of secondary structure was performed through Jpred (12) and the domain prediction was analyzed with UniProt (http://www.uniprot.org).

\section{Results}

Clinical information of the patient. A 69-year-old male patient was diagnosed with pleomorphic cell-type HCC with lymph node metastasis. The primary tumor was on the left lobe of the liver (10x8x6 cm in size) and showed invasive and septal cirrhosis with macro- and micronodules. It was a grade II to III HCC with prominent clear cell components. The results of serological tests for HBV showed that the tumor was positive for surface antigen, Anti-HBe (E) and core antibodies, indicating that the patient had chronic HBV infection.

Summary of exome sequencing. The mean total number of bases sequenced was $4.65 \mathrm{~Gb}$. The mean depth of the target region was 44 -fold. The mean percentage of the targeted bases which were covered at least once was $98.6 \%$. The mean percentage of the targeted bases which were covered sufficiently for variant calling (coverage $\geq 5$ ) was $92.1 \%$. The mean percentage of genes having $>95 \%$ of their coding bases called was $77.0 \%$. The details of the quality are shown in Table I.

Discovery of somatic variants. Raw sequence data revealed 1514 candidate somatic single nucleotide variations (SNVs) and 158 candidate somatic small insertions and deletions (InDels) in the HCC tissue and 63047 candidate germ-line SNVs and 4513 InDels. A series of subsequent qualifications of these data narrowed down these variants into 27 tumorspecific SNVs and seven InDels (Fig. 1). We focused our analysis on the six non-synonymous substitutions and one frameshift mutation affecting the integrity of the open reading frame (ORF). These seven candidate variants were located in different genes, SPATA21, PPCS, CDH12, OR1L3, PCK2, HUWE1 and PHF16. All the candidate variants were validated by PCR and sequencing, with the exception of a non-synonymous substitution in PPCS, which could not be distinguished from noise. Among these genes, HUWE1 and PHF16 were in the $\mathrm{X}$ chromosome. The details of the candidate variants are shown in Table II.

Bioinformatics analysis of validated variants. Six validated non-synonymous somatic variants were involved in the bioinformatics analysis, including five non-synonymous 
Table II. Non-synonymous somatic variants identified in HCC by whole-exome sequencing.

\begin{tabular}{llllll}
\hline Gene & Genomic locus & Accession no. & \multicolumn{1}{c}{ Mutation } & Protein & \multicolumn{1}{c}{ Function $^{\text {b }}$} \\
\hline SPATA21 & Chr1: 16748433 & NM_198546 & c.C68T & p.T23M & Calcium ion binding \\
PPCS & Chr1: 42922652 & NM_024664 & c.C416T & p.A139V & Phosphopantothenate- cysteine ligase activity \\
CDH12 & Chr5: 22078699 & NM_004061 & c.G87C & p.Q29H & Calcium ion binding \\
OR1L3 & Chr9: 125438256 & NM_001005234 & c.T848C & p.V283A & Odorant receptor \\
PCK2 & Chr14: 24566276 & NM_001018073 & c.G205A & p.E69K & GTP binding/kinase activity \\
HUWE1 & ChrX: 53596682 & NM_031407 & c.G6418A & p.A2140T & Acid - amino acid ligase activity/binding \\
PHF16 & ChrX: 46887461 & NM_001077445 & c.642_643insA & p.G214fs & Zinc ion binding
\end{tabular}

${ }^{\mathrm{a} C}$ Coordinates refer to the human reference genome hg 19 release. ${ }^{\mathrm{b}} \mathrm{Gene}$ Ontology (http://www.ebi.ac.uk/GOA/). HCC, hepatocellular carcinoma.

Table III. Bioinformatics analysis of validated variants.

\begin{tabular}{|c|c|c|c|c|c|c|c|}
\hline Gene & Mutation & Protein & $\begin{array}{l}\text { Chemistry } \\
\text { polarity }\end{array}$ & $\begin{array}{c}\text { Secondary structure } \\
\text { of protein }\end{array}$ & Domain & $\begin{array}{l}\text { PhyloP } \\
\text { value }\end{array}$ & $\begin{array}{l}\text { Conservative species } \\
\text { at protein level }\end{array}$ \\
\hline SPATA21 & c.C68T & P.T23M & Changed & Changed & Unknown & -2.074 & NA \\
\hline $\mathrm{CDH} 12$ & c.G87C & p.Q29H & Changed & Changed & Unknown & 2.801 & $\begin{array}{l}\text { Chimp, rhesus, cat, } \\
\text { mouse, chicken }\end{array}$ \\
\hline OR1L3 & c.T848C & p.V283A & NA & NA & Known & 1.866 & Gorilla \\
\hline PCK2 & c.G205A & p.E69K & Changed & Changed & Unknown & 5.208 & $\begin{array}{l}\text { Rhesus, cat, mouse, } \\
\text { fugu, chicken, } \\
\text { Xenopus, zebrafish, } \\
\text { C.elegans, Drosophila }\end{array}$ \\
\hline HUWE1 & c.G6418A & p.A2140T & Changed & Changed & Unknown & 5.451 & $\begin{array}{l}\text { Chimp, rhesus, mouse, } \\
\text { zebrafish, fugu }\end{array}$ \\
\hline PHF 16 & c.642_643insA & p.G214fs & Changed & Changed & Known & NA & NA \\
\hline
\end{tabular}

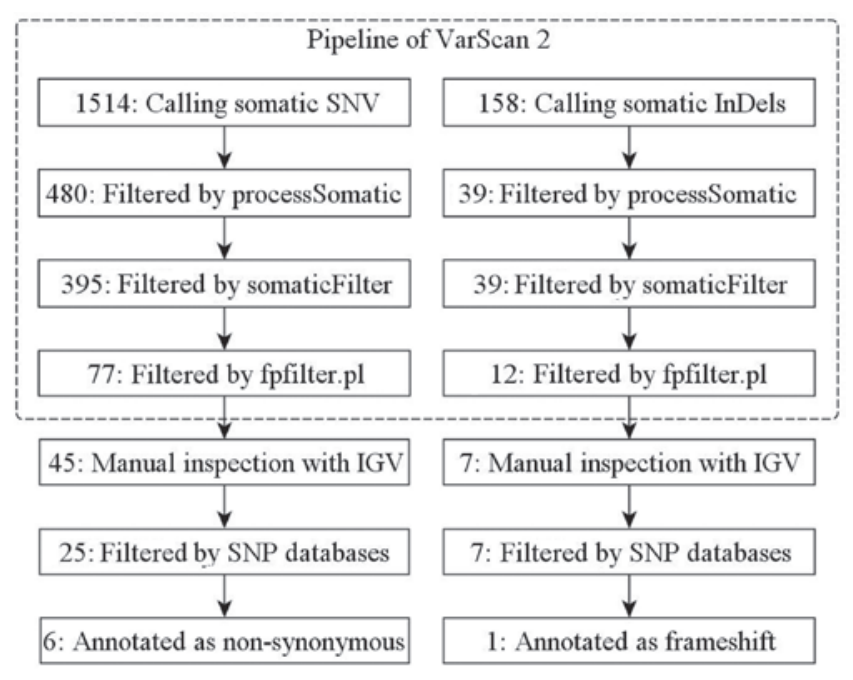

Figure 1. Processing of data obtained by whole-exome sequencing. The beginning of each box is the number of candidate somatic variants remaining after the step. SNV, single nucleotide variation; SNP, single nucleotide polymorphism; InDels, insertions and deletetions; IGV, Integrative Genomics Viewer.

substitutions and one InDel. The chemical polarity of all the variants was changed, with the exception of the variation in ORIL3. Five variants would affect the secondary structure of the protein and the variant of ORIL3 would not. The frameshift variation in $P H F 16$, located in the known domain, disturbed the gene structure. The results of conservation analysis indicated that the variants within $P C K 2$ and $H U W E 1$ may disturb the function of the protein encoded by these genes. The details of the analyzing of the candidate variants are shown in Table III.

\section{Discussion}

Using whole-exome sequencing, we identified seven non-synonymous somatic variants and validated six variants, which were previously unidentified in HCC. We further performed a bioinformatics analysis of the validated variants. The results suggest that the function of three mutated genes, PCK2, HUWE1 and PHF16, may be markedly changed.

HCC is a major health problem worldwide (13). Despite its global significance, liver cancer is understudied compared to other major lethal types of cancer, and few known genetic influences on the deveopment of HCC have been reported.

A number of studies in recent years have provided evidence that the $p 53$ tumor suppressor gene plays a major role in hepatocarcinogenesis (14). However, the frequency of $p 53$ variants and its mutation spectrum, with $75 \%$ missense variants, are exceptionally diverse in their position and nature, affecting over 200 codons scattered mainly 
throughout the central portion of the gene (15). There were no candidate somatic variants found within $p 53$ in the patient of the present study.

The insulin-like growth factor (IGF) signaling system is an essential regulator of growth and development (16). The biological actions of the axis comprise a complex network of molecules whose main components are two high affinity mitogenic ligands: IGF1 and IGF2. The type 1 IGF receptor (IGF1R) has tyrosine kinase activity, the type 2 IGF receptor (IGF2R) is involved in the internalization and degradation of IGF2 and at least six high-affinity IGF-binding proteins (IGFBPs), which modulate the amount and bioactivity of locally available IGFs. Despite its role in normal physiology, the IGF axis is involved in the pathogenesis of several human malignancies, including breast, colon, prostate, lung and liver cancer (17). In HCC, the most frequently described aberrant feature concerning this pathway is overexpression of IGF2, which has been found in preneoplastic lesions (18). This mitogen, highly expressed during embryonic development, is markedly downregulated after birth by tight epigenetic regulation of the P2-P4 fetal promoters. Reactivation of IGF2 expression involves loss of specific imprinting and hypomethylation (19). Allelic losses of IGF2R has been detected in $60-70 \%$ of HCC cases, with inactivating variants in the remaining allele also reported (20). In addition, reduced expression of IGFBP-3 associated with promoter hypermethylation has been reported in human HCC samples (21). A recent study found aberrant activation of IGF1R in $21 \%$ of early stage hepatitis C-related HCC xases, and provided preclinical evidence of antineoplastic activity following IGF1R selective blockade using a monoclonal antibody (22). The potential role of the HBx viral protein as an inducer of IGF-IR expression has also been suggested (23). In the present study, the candidate gene $P C K 2$ is in the insulin signaling pathway. PCK2 encodes a member of the phosphoenolpyruvate carboxykinase (GTP) family. The protein is a mitochondrial enzyme that catalyzes the conversion of oxaloacetate to phosphoenolpyruvate in the presence of GTP. A cytosolic form encoded by a different gene has also been characterized and is the key enzyme of gluconeogenesis in the liver. The encoded protein may serve a similar function, although it is constitutively expressed and not modulated by the hormones, including glucagon and insulin, that regulate the cytosolic form. Hill et al reported that the direct action of TNF to decrease the PEPCK transcription rate was confirmed in vitro with H-4-II-E Reuber hepatoma cells (24). The authors suggested that $P C K 2$ may be associated with hepatocarcinogenesis.

CTNNB1 and AXIN1 variants are frequently found in HCC (25). Variants prevent $\beta$-catenin ubiquitination and subsequent degradation. Nuclear accumulation of $\beta$-catenin induces the transcription of several genes associated with cell differentiation and proliferation. In our study, the X-linked candidate gene HUWE1 is in the ubiquitin-mediated proteolysis pathway. The mutated gene may prevent $\beta$-catenin ubiquitination and subsequent degradation, similar to CTNNBI and AXIN1. Notably, it is the X-linked gene which may contribute to the higher incidence of HCC in males.

In conclusion, we identified six genes with non-synonymous somatic variants in a HCC patient. Some of these genes are involved in pathways associated with cell proliferation and differentiation, which is known to be important in hepatocarcinogenesis. Our results indicate that the insulin signaling and ubiquitin-mediated proteolysis pathways may be essential to HCC, and their relevant gene signature may be a target for new therapies in HCC. Larger sample sizes are needed to confirm or disprove our hypothesis.

\section{Acknowledgements}

We thank Dr Li Tong for the suggestions about the experiment design. This study was supported by the Provincial Education Science Foundation of Henan (2009A330004).

\section{References}

1. Motola-Kuba D, Zamora-Valdés D, Uribe M and MéndezSánchez N: Hepatocellular carcinoma. An overview. Ann Hepatol 5: 16-24, 2006

2. Srivatanakul P, Sriplung H and Deerasamee S: Epidemiology of liver cancer: an overview. Asian Pac J Cancer Prev 5: 118-125, 2004.

3. McGlynn KA and London WT: Epidemiology and natural history of hepatocellular carcinoma. Best Pract Res Clin Gastroenterol 19: 3-23, 2005.

4. Clark JM: The epidemiology of nonalcoholic fatty liver disease in adults. J Clin Gastroenterol 40 (Suppl 1): S5-S10, 2006.

5. Teo EK and Fock KM: Hepatocellular carcinoma: an Asian perspective. Dig Dis 19: 263-268, 2001.

6. Li H and Durbin R: Fast and accurate long-read alignment with Burrows-Wheeler transform. Bioinformatics 26: 589-595, 2010.

7. Li H, Handsaker B, Wysoker A, et al; 1000 Genome Project Data Processing Subgroup: The Sequence Alignment/Map format and SAMtools. Bioinformatics 25: 2078-2079, 2009.

8. Koboldt DC, Zhang Q, Larson DE, et al: VarScan 2: somatic mutation and copy number alteration discovery in cancer by exome sequencing. Genome Res 22: 568-576, 2012.

9. Robinson JT, Thorvaldsdóttir H, Winckler W, et al: Integrative genomics viewer. Nat Biotechnol 29: 24-26, 2011.

10. Pollard KS, Hubisz MJ, Rosenbloom KR and Siepel A: Detection of nonneutral substitution rates on mammalian phylogenies. Genome Res 20: 110-121, 2010.

11. Schwarz JM, Rödelsperger C, Schuelke M and Seelow D: MutationTaster evaluates disease-causing potential of sequence alterations. Nat Methods 7: 575-576, 2010.

12. Cole C, Barber JD and Barton GJ: The Jpred 3 secondary structure prediction server. Nucleic Acids Res 36: W197-W201, 2008.

13. Llovet JM, Di Bisceglie AM, Bruix J, et al; Panel of Experts in HCC-Design Clinical Trials: Design and endpoints of clinical trials in hepatocellular carcinoma. J Natl Cancer Inst 100: 698-711, 2008

14. Edamoto Y, Hara A, Biernat W, et al: Alterations of RB1, p53 and Wnt pathways in hepatocellular carcinomas associated with hepatitis $\mathrm{C}$, hepatitis $\mathrm{B}$ and alcoholic liver cirrhosis. Int $\mathrm{J}$ Cancer 106: 334-341, 2003.

15. Hainaut $P$ and Hollstein M: $\mathrm{p} 53$ and human cancer: the first ten thousand mutations. Adv Cancer Res 77: 81-137, 2000.

16. Pollak M: Insulin and insulin-like growth factor signalling in neoplasia. Nat Rev Cancer 8: 915-928, 2008.

17. Sachdev D and Yee D: Disrupting insulin-like growth factor signaling as a potential cancer therapy. Mol Cancer Ther 6: 1-12, 2007.

18. Laurent-Puig P and Zucman-Rossi J: Genetics of hepatocellular tumors. Oncogene 25: 3778-3786, 2006.

19. Tang SH, Yang DH, Huang W, Zhou HK, Lu XH and Ye G: Hypomethylated P4 promoter induces expression of the insulinlike growth factor-II gene in hepatocellular carcinoma in a Chinese population. Clin Cancer Res 12: 4171-4177, 2006.

20. De Souza AT, Hankins GR, Washington MK, Orton TC and Jirtle RL: M6P/IGF2R gene is mutated in human hepatocellular carcinomas with loss of heterozygosity. Nat Genet 11: 447-449, 1995. 
21. Hanafusa T, Yumoto Y, Nouso K, et al: Reduced expression of insulin-like growth factor binding protein-3 and its promoter hypermethylation in human hepatocellular carcinoma. Cancer Lett 176: 149-158, 2002.

22. Tovar V, Alsinet C, Villanueva A, et al: IGF activation in a molecular subclass of hepatocellular carcinoma and pre-clinical efficacy of IGF-1R blockage. J Hepatol 52: 550-559, 2010.

23. Kim SO, Park JG and Lee YI: Increased expression of the insulin-like growth factor I (IGF-I) receptor gene in hepatocellular carcinoma cell lines: implications of IGF-I receptor gene activation by hepatitis B virus X gene product. Cancer Res 56 : 3831-3836, 1996.
24. Hill MR and McCallum RE: Identification of tumor necrosis factor as a transcriptional regulator of the phosphoenolpyruvate carboxykinase gene following endotoxin treatment of mice. Infect Immun 60: 4040-4050, 1992.

25. Villanueva A, Newell P, Chiang DY, Friedman SL and Llovet JM: Genomics and signaling pathways in hepatocellular carcinoma. Semin Liver Dis 27: 55-76, 2007. 